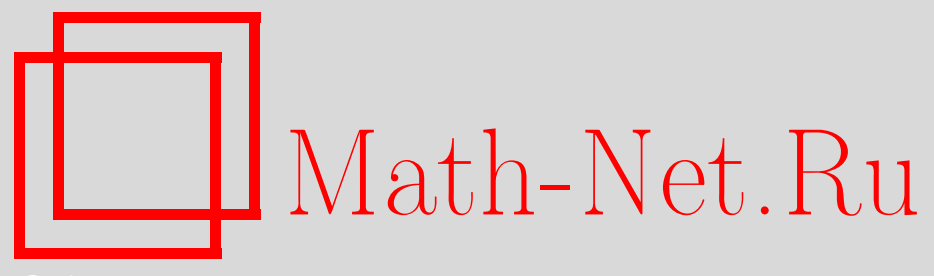

В. В. Жиков, С. Е. Пастухова, О свойстве повышенной суммируемости для параболических систем переменного порядка нелинейности, Матем. заметки, 2010, том 87, выпуск 2, 179-200

DOI: https://doi.org/10.4213/mzm5256

Использование Общероссийского математического портала Math-Net.Ru подразумевает, что вы прочитали и согласны с пользовательским соглашением http://www . mathnet.ru/rus/agreement

Параметры загрузки:

IP: 3.81 .55 .215

26 апреля 2023 г., 18:31:27

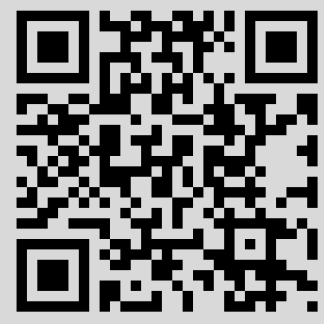


Том 87 выпуск 2 февраль 2010

УДК 517.95

\section{О свойстве повышенной суммируемости для параболических систем переменного порядка нелинейности}

\section{В. В. Жиков, С. Е. Пастухова}

Изучается параболическая система вида $\partial_{t} u=\operatorname{div}_{x} A\left(x, t, \nabla_{x} u\right)$ в ограниченном цилиндре $Q_{T}=\Omega \times(0, T) \subset \mathbb{R}_{x, t}^{n+1}$. Здесь матричная функция $A(x, t, \xi)$ подчинена по переменной $\xi$ степенным условиям роста и коэрцитивности с переменным показателем $p(x, t)$. Предполагается, что $p(x, t)$ имеет логарифмический модуль непрерывности и удовлетворяет оценке

$$
\frac{2 n}{n+2}<\alpha \leqslant p(x, t) \leqslant \beta<\infty .
$$

Для слабого решения системы получены внутри цилиндра $Q_{T}$ оценки повышенной суммируемости градиента. Основу метода составляют специального рода локализация и адаптированный для параболических задач локальный вариант леммы Геринга с переменным показателем суммируемости, доказываемый в работе.

Библиография: 10 названий.

\section{1. Введение}

1.1. Постановка задачи и основной результат. Изучается регулярность решений параболической системы

$$
\partial_{t} u_{i}=\operatorname{div}_{x} A_{i}(x, t, \nabla u), \quad i=1, \ldots, N, \quad(x, t) \in \Omega \times(0, T)=Q_{T},
$$

где $\Omega$ - область в $\mathbb{R}^{n}, 0<T<\infty, u=\left(u_{1}, \ldots, u_{N}\right)$ - вектор решения, $\nabla u=\left\{\partial_{x_{j}} u_{i}\right\}-$ матрица градиента. Вектор-функция

$$
A_{i}(x, t, \xi): \Omega \times(0, T) \times \mathbb{R}^{n \times N} \rightarrow \mathbb{R}^{n}
$$

предполагается каратеодориевой, т.е. измеримой по $x, t$ для всех $\xi$ и непрерывной по $\xi$ для п.в. $x, t$. Кроме того, $A_{i}(x, t, \xi)$ подчинена следующему структурному условию.

Работа выполнена при поддержке Российского фонда фундаментальных исследований (грант № 08-01-9907-a). 
Условие (S). Пусть

$$
p=p(x, t) \in C\left(Q_{T}\right), \quad \frac{2 n}{n+2}<\alpha \leqslant p(x, t) \leqslant \beta<\infty, \quad(x, t) \in Q_{T},
$$

и пусть существуют константы $c_{i}>0, i=0,1,2$, такие, что

$$
\begin{gathered}
\sum_{i=1}^{N} A_{i}(x, t, \xi) \cdot \xi_{i} \geqslant c_{0}|\xi|^{p}-h_{0}, \\
\left|A_{i}(x, t, \xi)\right| \leqslant c_{1}|\xi|^{p-1}+h_{1}, \quad i=1, \ldots, N,
\end{gathered}
$$

для п.в. $(x, t) \in Q_{T}$, где $h_{0}, h_{1}$ - заданные на $Q_{T}$ измеримые функции такие, что

$$
\left\|\left|h_{1}\right|^{p /(p-1)}+\left|h_{0}\right|\right\|_{L^{q}\left(Q_{T}\right)}<c_{2}, \quad q>1 .
$$

Здесь $\xi_{1}, \ldots, \xi_{N}-$ столбцы матрицы $\xi$.

Условию $(\mathrm{S})$ удовлетворяет система с $p(x, t)$-лапласианом

$$
\partial_{t} u_{i}=\operatorname{div}_{x}\left(|\nabla u|^{p(x, t)-2} \nabla u_{i}+g_{i}\right), \quad i=1, \ldots, N,
$$

если $|g|^{p /(p-1)} \in L^{q}\left(Q_{T}\right)$ для некоторого $q>1$.

Далее показатель $p(x, t)$ имеет логарифмический модуль непрерывности:

$$
\begin{aligned}
&\left|p(x, t)-p\left(x^{\prime}, t^{\prime}\right)\right| \leqslant \frac{1}{\ln \left(1 /\left|x-x^{\prime}\right|+1 /\left|t-t^{\prime}\right|\right)}, \\
&(x, t),\left(x^{\prime}, t^{\prime}\right) \in Q_{T}, \quad\left|x-x^{\prime}\right|+\left|t-t^{\prime}\right| \leqslant \frac{1}{2} .
\end{aligned}
$$

Введем класс функций

$$
W=W\left(Q_{T}\right)=\left\{u(x, t): u \in L^{2}\left(Q_{T}\right)^{N},|\nabla u|^{p} \in L^{1}\left(Q_{T}\right)\right\} .
$$

Наделенный естественной нормой он становится банаховым пространством. Будем называть его параболическим пространством Соболева-Орлича с переменным показателем $p$.

Под слабым решением системы (1.1) понимаем функцию $u \in W$, для которой уравнения (1.1) выполнены в смысле распределений.

Теорема 1.1. Если и - слабое решение системы (1.1), то существует $\delta>0$ maкое, чmo

$$
\left\||\nabla u|^{p+\delta}\right\|_{L^{1}\left(Q^{\prime}\right)} \leqslant C<\infty, \quad Q^{\prime} \Subset Q_{T} .
$$

Постоянная $\delta$ зависит лишь от $n$ и констант $c_{0}, c_{1}, q, \beta$ из условия $(\mathrm{S})$, а постоянная $C$ - от тех же величин, а также от $N, c_{2},\left\||\nabla u|^{p}\right\|_{L^{1}\left(Q_{T}\right)} u Q^{\prime}$.

Как следствие, слабое решение локально внутри $Q_{T}$ принадлежит пространству Соболева-Орлича с показателем, превышающим показатель энергетического пространства. Еще большим, благодаря теоремам вложения, оказывается показатель суммируемости самого решения.

При $p=$ const $>2 n /(n+2)$ теорема 1.1 доказана в [1]. Скалярное уравнение $(1.2)$ (т.е. $N=1$ ) изучалось в [2], где специфика скалярной задачи оказалась весьма существенной, причем охвачен был фактически лишь случай $p(x, t) \geqslant 2$. 
1.2. Теоремы о плотности и сглаживание. Данное выше определение слабого решения системы (1.1) допускает (как и в случае постоянного $p$, см. [3], [4]) несколько эквивалентных формулировок. Укажем те из них, которые наиболее удобны для вывода интересующих нас оценок.

ПреДлОЖеНИЕ 1.2. Для функиии $u \in W$ выполнены в смысле распределений уравнения (1.1) тогда и только тогда, когда для любой функиии $\zeta \in C^{\infty}\left(0, T ; C_{0}^{\infty}(\Omega)^{N}\right)$ справедливо интегральное тождество

$$
\left.\int_{\Omega} u \cdot \zeta d x\right|_{t_{1}} ^{t_{2}}+\int_{t_{1}}^{t_{2}} \int_{\Omega}\left(-u \cdot \partial_{t} \zeta+\sum_{i} A_{i} \cdot \nabla \zeta_{i}\right) d x d t=0 \quad \text { для п.в. } t_{1}, t_{2} \in(0, T) .
$$

Встает вопрос о расширении множества пробных функций в (1.5), прежде всего, в связи с методом доказательства. Поясним это обстоятельство подробнее.

Доказательство свойства повышенной суммируемости (1.4) следует адаптированной к параболическим задачам методике, предложенной Модика и Джаквинта при изучении эллиптических уравнений, см. [5], [6]. Эта методика заключается в том, что сначала доказывается параболическое неравенство Каччопполи, а затем из него с помощью соответствующих неравенств Соболева-Пуанкаре выводится для градиента решения так называемое обратное неравенство Гёльдера с разными носителями, что позволяет на заключительной стадии применить теорему типа классической леммы Геринга [7]. Все перечисленные утверждения в параболической ситуации с переменным порядком суммируемости нетрадиционны, имеют усложненную форму и требуют дополнительных усилий. Этот весьма длинный и многоэтапный путь начинается с первого шага - подстановки в тождество (1.5) подходящей пробной функции. Таковой в идеале, при достаточной регулярности решения, могла бы стать функция $\psi u$ - произведение самого решения $u$ на специально подобранную срезку $\psi$. К сожалению, в общих предположениях решение недостаточно регулярно, прежде всего по переменной $t$, и на некоторых этапах приходится заменять решение регулярными аппроксимациями, например, сглаживаниями.

Уточним определение операции сглаживания: для $f \in L^{1}\left(Q_{T}\right)$ полагаем

$$
I^{h} f=f_{h}(x, t) \equiv h^{-(n+1)} \int_{\mathbb{R}^{n+1}} \rho\left(\frac{x-x^{\prime}}{h}, \frac{t-t^{\prime}}{h}\right) f\left(x^{\prime}, t^{\prime}\right) d x^{\prime} d t^{\prime},
$$

где $\rho(x, t)$ - гладкое неотрицательное финитное четное ядро сглаживания такое, что $\int_{\mathbb{R}^{n+1}} \rho d x d t=1$, и $h$ - малый положительный параметр. Ввиду четности ядра сглаживания выполнено равенство

$$
\int_{Q_{T}} f_{h} g d x d t=\int_{Q_{T}} f g_{h} d x d t
$$

для любых функций $f, g \in L^{2}\left(Q_{T}\right)$, одна из которых имеет компактный в $Q_{T}$ носитель.

Сглаживание находит широкое применение в параболической теории с постоянным показателем $p$, см. [3], [4], благодаря следующим свойствам.

Tеорема 1.3. Если $f \in L^{\gamma}\left(Q_{T}\right)$, где $\gamma \geqslant 1$, то при $h \rightarrow 0$ uмеет место сходимость $f_{h} \rightarrow f$ в $L^{\gamma}\left(Q^{\prime}\right)$ для любого $Q^{\prime}=\Omega^{\prime} \times\left(t_{1}, t_{2}\right) \Subset Q_{T} u f_{h}(\cdot, t) \rightarrow f(\cdot, t)$ в $L^{\gamma}\left(\Omega^{\prime}\right)$ для п.в. $t \in(0, T)$ и любой $\Omega^{\prime} \Subset \Omega$. 
Здесь мы имеем дело с обычными пространствами Лебега $L^{\gamma}\left(Q_{T}\right)$, которым приписан постоянный порядок $\gamma$. Их аналогами "переменного порядка" будут пространства Орлича $L^{p(\cdot)}\left(Q_{T}\right)$ (см. определение в разделе 7 ), естественно возникающие при изучении уравнений с переменным порядком нелинейности. Для нас будет важна

ТЕОРема 1.4. При выполнении логарифмического условия (1.3) имеет место сходимость $f_{h} \rightarrow f$ в $L^{p(\cdot)}\left(Q^{\prime}\right), Q^{\prime} \Subset Q_{T}$, при $h \rightarrow 0$ для любой функиии $f \in$ $L^{p(\cdot)}\left(Q_{T}\right)$.

Ввиду равенства $(\nabla f)_{h}=\nabla(f)_{h}$ из теорем 1.3 и 1.4 (а также свойств сходимости в $L^{p(\cdot)}\left(Q_{T}\right)$, см. раздел 7$)$ следует

ТЕорема 1.5. При выполнении логарифмического условия (1.3), если $f \in W\left(Q_{T}\right)$ $u Q^{\prime} \Subset Q_{T}$, mo $\int_{Q^{\prime}}\left(\left|f-f_{h}\right|^{2}+\left|\nabla f-\nabla f_{h}\right|^{p}\right) d x d t \rightarrow 0$ nрu $h \rightarrow 0$.

Коротко о доказательстве самой теоремы 1.4. Ключевым в нем оказывается установленный в [8] факт об ограниченности оператора сглаживания $I^{h}$ в пространствах Орлича. Для полноты изложения мы даем вывод этого утверждения в разделе 7 . Там же приведены другие необходимые для нас сведения из теории пространств Орлича и Соболева-Орлича.

В терминах сглаживания формулируется еще одно тождество для слабого решения системы (1.1), полезное в дальнейшем:

$$
\int_{t_{1}}^{t_{2}} \int_{\Omega}\left(\partial_{t} u_{h} \cdot \zeta+\sum_{i}\left(A_{i}\right)_{h} \cdot \nabla \zeta_{i}\right) d x d t=0, \quad \zeta \in C^{\infty}\left(0, T ; C_{0}^{\infty}(\Omega)^{N}\right), \quad 0<t_{1}<t_{2}<T
$$

Для вывода (1.7) запишем по определению слабого решения тождество на финитных функциях

$$
\int_{Q_{T}}\left(-u \cdot \partial_{t} \zeta+\sum_{i} A_{i} \cdot \nabla \zeta_{i}\right) d x d t=0, \quad \zeta \in C_{0}^{\infty}\left(Q_{T}\right)^{N}
$$

и возьмем в качестве пробной функции сглаживание $\zeta=\eta_{h}$, где $\eta \in C_{0}^{\infty}\left(Q_{T}\right)^{N}$. Тогда по свойствам сглаживания

$$
\int_{Q_{T}}\left(\partial_{t} u_{h} \cdot \eta+\sum_{i}\left(A_{i}\right)_{h} \cdot \nabla \eta_{i}\right) d x d t=0, \quad \eta \in C_{0}^{\infty}\left(Q_{T}\right)^{N}
$$

Теперь выберем функцию $\eta$ специальным образом, используя $\zeta$ из (1.7),

$$
\eta(x, t)=\zeta(x, t) \chi_{k}(t), \quad \chi_{k} \in C_{0}^{\infty}(0, T),
$$

где $\chi_{k}=0$ вне $\left(t_{1}, t_{2}\right), \chi_{k}=1$ на $\left(t_{1}+1 / k, t_{2}-1 / k\right), \chi_{k}$ монотонна на $\left(t_{1}, t_{1}+1 / k\right)$ и $\left(t_{2}-1 / k, t_{2}\right)$. Переход к пределу при $k \rightarrow \infty$ в тождестве (1.8) с пробной функцией $\eta$ вида (1.9) дает (1.7).

Далее повсеместно используем следующие обозначения:

- $z=(x, t), z_{0}=\left(x_{0}, t_{0}\right)$ - координаты точки в $\mathbb{R}^{n+1}$;

- $B_{r}\left(x_{0}\right)$ - шар в $\mathbb{R}^{n}$ радиуса $r$ с центром в точке $x_{0}$;

- $Q_{r, \tau}\left(z_{0}\right)=B_{r}\left(x_{0}\right) \times\left(t_{0}-\tau, t_{0}+\tau\right)$ - цилиндр в $\mathbb{R}^{n+1}$ с центром в точке $z_{0}$;

- $|E|-m$-мерная мера Лебега $m$-мерного измеримого множества $E$;

- $f_{E} \cdot=(1 /|E|) \int_{E} \cdot-$ среднее по множеству $E$;

- $\omega_{n}$ - объем единичного шара в $\mathbb{R}^{n}$. 


\section{2. Выбор геометрических параметров}

В нашем методе доказательства повышенной суммируемости градиента решения системы (1.1) (см. краткое описание в п. 1.2) один из важнейших моментов, он же и начальный, - выбор подходящей срезающей функции, иными словами, специального рода локализация. Эта локализация проводится в малых цилиндрах $Q_{r, \tau}\left(z_{0}\right)$, $z_{0} \in Q_{T}$, со специально подобранными геометрическими параметрами $r$ и $\tau$. Подбирая параметры $r$ и $\tau$, мы преследуем, прежде всего, две цели. Во-первых, компенсируем неоднородность параболического оператора порядка $p>1$. Напомним, что показатель $p=p(z)$ переменный и меняется в любом диапазоне $[\alpha, \beta] \subset(2 n /(n+2), \infty)$. Во-вторых, мы добиваемся некоторой подчиненности определенных интегральных средних (см. (2.7)), что существенно помогает при выводе локальных оценок. Специальный выбор геометрических параметров мы описываем в общей ситуации для произвольного параболического цилиндра и заданной в нем функции. Далее, изучая решение $u$ системы (1.1), саму эту функцию мы строим подходящим образом, включая в нее градиент $\nabla u$ и функции $h_{0}$ и $h_{1}$ из условия $(\mathrm{S})$. Отметим, что отделенность показателя $p(z)$ от нижней границы $2 n /(n+2)$ используется в наших построениях постоянно.

Пусть $Q$ - цилиндр в $\mathbb{R}^{n+1}$ и непрерывный в $\bar{Q}$ показатель $p(z)$ удовлетворяет условию

$$
\frac{2 n}{n+2}<\alpha \leqslant p(z) \leqslant \beta<\infty, \quad z \in \bar{Q}
$$

Рассмотрим $f \geqslant 0$ такую, что $f^{p} \in L^{1}(Q)$. Далее обозначаем через $d(z)$ параболическое расстояние от точки $z$ до границы $\partial Q$. Напомним, что параболическое расстояние между точками $z=(x, t), z^{\prime}=\left(x^{\prime}, t^{\prime}\right)$ есть $\max \left\{\left|x-x^{\prime}\right|,\left|t-t^{\prime}\right|^{1 / 2}\right\}$. Удобно считать цилиндр $Q$ достаточно малым, так что $\left.d(z)\right|_{Q} \leqslant 1$.

Случай $p_{0}=p\left(z_{0}\right) \geqslant 2$. Определим константу $\lambda_{0}>1$ равенством $\int_{Q} f^{p} d z+1=\lambda_{0}^{2}$ и введем функции

$$
F(z)=f(z) \rho(z) \widetilde{c}^{-1}, \quad \rho(z)=d(z)^{(n+2) / 2},
$$

где константа $\widetilde{c}>1$ уточнена позже. Пусть $F\left(z_{0}\right)>\lambda \geqslant \lambda_{0}$, т.е. $f\left(z_{0}\right)>\widetilde{c} \widetilde{\lambda}$, где $\widetilde{\lambda}=\lambda / \rho_{0}$ и $\rho_{0}=\rho\left(z_{0}\right)$. Положим $\theta=\widetilde{\lambda}^{2-p_{0}}$ и будем рассматривать цилиндры $Q_{r}=Q_{r, \theta r^{2}}\left(z_{0}\right)$. Поскольку $0<\theta \leqslant 1$ (так как $\left.\widetilde{\lambda} \geqslant 1, p_{0} \geqslant 2\right)$, при $r \in\left[d\left(z_{0}\right) / 16, d\left(z_{0}\right)\right)$ имеем $Q_{r} \subset Q$ и для достаточно большой константы $\widetilde{c}\left(\widetilde{c} \geqslant 16^{n+2} / 2 \omega_{n}\right)$

$$
f_{Q_{r}} f^{p} d z \leqslant \frac{1}{2 \omega_{n} \theta r^{n+2}} \int_{Q} f^{p} d z \leqslant \frac{\lambda^{p_{0}-2} \rho_{0}^{2-p_{0}} \lambda_{0}^{2}}{2 \omega_{n} r^{n+2}} \leqslant\left(\frac{\lambda}{\rho_{0}}\right)^{p_{0}} \widetilde{c}^{p_{0}}=(\widetilde{c} \tilde{\lambda})^{p_{0}} .
$$

Вспомним, что $f\left(z_{0}\right)>\widetilde{c} \widetilde{\lambda}$ и по теореме Лебега $\lim _{r \rightarrow 0} f_{Q_{r}} f^{p} d z>(\widetilde{c} \widetilde{\lambda})^{p_{0}}$ для п.в. $z_{0}$. Тогда из непрерывности интеграла $f_{Q_{r}} f^{p} d z$ по $r$ следует существование такого $r=$ $r_{z_{0}} \in\left(0, d\left(z_{0}\right) / 16\right)$, что

$$
(\widetilde{c} \widetilde{\lambda})^{p_{0}}=f_{Q_{r}} f^{p} d z>f_{Q_{R}} f^{p} d z \quad \text { для любого } \quad R \in\left(r, d\left(z_{0}\right)\right) .
$$


Случай $p_{0}=p\left(z_{0}\right)<2$. Определим константу $\lambda_{0}>1$ равенством $\int_{Q} f^{p} d z+1=$ $\lambda_{0}^{(\alpha(n+2)-2 n) / 2}$ и введем функции

$$
F(z)=f(z) \rho(z) \widetilde{c}^{-1}, \quad \rho(z)=d(z)^{\sigma(z)}, \quad \sigma(z)=\frac{2(n+2)}{p(n+2)-2 n},
$$

где константа $\widetilde{c}>1$ та же, что ранее. Рассмотрим цилиндры

$$
Q_{\gamma}=Q_{\widetilde{\lambda}^{\left(p_{0}-2\right) / 2} \gamma, \gamma^{2}}\left(z_{0}\right), \quad \widetilde{\lambda}=\frac{\lambda}{\rho_{0}} .
$$

Если $\gamma \in\left[d\left(z_{0}\right) / 16, d\left(z_{0}\right)\right)$, то $Q_{\gamma} \subset Q\left(\right.$ так как $\left.\widetilde{\lambda}>1, p_{0}<2\right)$ и

$$
\begin{aligned}
f_{Q_{\gamma}} f^{p} d z & \leqslant \frac{1}{\left|Q_{\gamma}\right|} \int_{Q} f^{p} d z \leqslant\left(\frac{\lambda}{\rho_{0}}\right)^{\left(2-p_{0}\right) n / 2} \frac{\lambda_{0}^{\left(p_{0}(n+2)-2 n\right) / 2}}{2 \omega_{n} \gamma^{n+2}} \\
& \leqslant\left(\frac{\lambda}{\rho_{0}}\right)^{p_{0}} \frac{\rho_{0}^{\left(p_{0}-2\right) n / 2+p_{0}}}{2 \omega_{n} \gamma^{n+2}}=\frac{\widetilde{\lambda}^{p_{0}}}{2 \omega_{n}}\left(\frac{d\left(z_{0}\right)}{\gamma}\right)^{n+2} \leqslant(\widetilde{c} \widetilde{\lambda})^{p_{0}} .
\end{aligned}
$$

Пусть $F\left(z_{0}\right)>\lambda$, т.е. $f\left(z_{0}\right)>\widetilde{c} \widetilde{\lambda}$. Тогда те же соображения непрерывности, что и раньше, приводят к существованию для п.в. $z_{0}$ такого $\gamma_{0} \in\left(0, d\left(z_{0}\right) / 16\right)$, что

$$
(\widetilde{c} \widetilde{\lambda})^{p_{0}}=f_{Q_{\gamma_{0}}} f^{p} d z>f_{Q_{\gamma}} f^{p} d z \quad \text { для любого } \quad \gamma \in\left(\gamma_{0}, d\left(z_{0}\right)\right) .
$$

Положим $r=\widetilde{\lambda}^{\left(p_{0}-2\right) / 2} \gamma_{0}, \theta=\widetilde{\lambda}^{2-p_{0}}$. Тогда $Q_{\gamma_{0}}=Q_{\widetilde{\lambda}^{\left(p_{0}-2\right) / 2} \gamma_{0}, \gamma_{0}^{2}}\left(z_{0}\right)=Q_{r, \theta r^{2}}\left(z_{0}\right)$, и можно придать единую форму неравенствам $(2.3),(2.5)$, равно как соотношениям (2.2), (2.4), если ввести

$$
F(z)=f(z) \rho(z) \widetilde{c}^{-1}, \quad \rho(z)=d(z)^{\sigma(z)}, \quad \sigma(z)= \begin{cases}\frac{n+2}{2} & \text { при } p(z) \geqslant 2, \\ \frac{2(n+2)}{p(n+2)-2 n} & \text { при } p(z)<2 .\end{cases}
$$

Лемма 2.1. Для некоторых констант $\widetilde{c}=\widetilde{c}(n)>1 u \lambda_{0}=\lambda_{0}\left(n, \alpha,\left\|f^{p}\right\|_{L^{1}(Q)}\right)>1$ и для п.в. $z_{0}$, удовлетворяющих условию

$$
f\left(z_{0}\right)>\widetilde{c} \widetilde{\lambda}, \quad \text { где } \quad \tilde{\lambda}=\frac{\lambda}{\rho_{0}}, \quad \lambda \geqslant \lambda_{0}, \quad \rho_{0}=\rho\left(z_{0}\right)
$$

(см. $\rho(z)$ в (2.6)), найдется $r=r_{z_{0}} \in\left(0, d\left(z_{0}\right) / 16\right)$ такое, что

$$
(\widetilde{c} \widetilde{\lambda})^{p_{0}}=f_{Q_{r, \theta r^{2}}\left(z_{0}\right)} f^{p} d z>f_{Q_{R, \theta R^{2}}\left(z_{0}\right)} f^{p} d z, \quad \theta=\widetilde{\lambda}^{2-p_{0}}, \quad r<R<d\left(z_{0}\right) .
$$

Введем цилиндры

$$
Q^{i}=Q_{2^{i} r, 2^{2 i} \theta r^{2}}\left(z_{0}\right), \quad i=0,1,2,3,
$$

с такими же параметрами $r$ и $\theta$, как в (2.7). Справедлива 
Лемма 2.2. Пусть $z_{0}, \lambda, r=r\left(z_{0}, \lambda\right), \rho(z)$ те же, что в лемме 2.1. Тогда найдутся константы $k_{i}(n)>1$ такие, что

$$
k_{i}^{-1} \widetilde{\lambda}^{p_{0}} \leqslant f_{Q^{i}} f^{p} d z \leqslant k_{i} \widetilde{\lambda}^{p_{0}}, \quad \tilde{\lambda}=\frac{\lambda}{\rho\left(z_{0}\right)}, \quad \theta=\widetilde{\lambda}^{2-p_{0}}, \quad i=0,1,2,3 .
$$

ДоказАтельство. Очевидно неравенство

$$
f_{Q^{i+1}} f^{p} d z \geqslant \frac{\left|Q^{i}\right|}{\left|Q^{i+1}\right|} f_{Q^{i}} f^{p} d z=2^{-(n+2)} f_{Q^{i}} f^{p} d z, \quad i=0,1,2,
$$

которое вместе с (2.7) дает соотношения

$$
\begin{aligned}
c_{0}^{3}(\widetilde{c} \tilde{\lambda})^{p_{0}} & \leqslant c_{0}^{2} f_{Q^{1}} f^{p} d z \leqslant c_{0} f_{Q^{2}} f^{p} d z \\
& \leqslant f_{Q^{3}} f^{p} d z<f_{Q^{0}} f^{p} d z=(\widetilde{c} \widetilde{\lambda})^{p_{0}}, \quad c_{0}=2^{-(n+2)},
\end{aligned}
$$

откуда следует (2.9).

ЗАмечАниЕ. Свойства (2.7) и (2.9), которыми обладают цилиндры вида (2.8), налагают связи между параметрами $r$ и $\widetilde{\lambda}$. В частности,

$$
\begin{gathered}
\widetilde{\lambda}^{p_{0}} \leqslant \frac{1}{\left|Q^{0}\right|}\left\|f^{p}\right\|_{L^{1}(Q)}=\frac{c}{r^{n+2} \widetilde{\lambda}^{2-p_{0}}}, \quad \widetilde{\lambda}^{2} \leqslant \frac{c}{r^{n+2}}, \quad r \leqslant c \widetilde{\lambda}^{-2 /(n+2)}, \\
\theta r^{2}=\widetilde{\lambda}^{2-p_{0}} r^{2} \leqslant c^{2} \widetilde{\lambda}^{2-p_{0}-4 /(n+2)}=c^{2} \widetilde{\lambda}^{2 n /(n+2)-p_{0}}, \quad c=c\left(n,\left\|f^{p}\right\|_{L^{1}(Q)}\right),
\end{gathered}
$$

и благодаря нижней оценке $(2.1)$, за счет выбора достаточно больших $\widetilde{\lambda}$ как угодно малы $r$ и $\theta r^{2}$. Как следствие, если $\omega(s)=\kappa / \ln (1 / s), \kappa>0$, то

$$
\widetilde{\lambda}^{\omega\left(r+\theta r^{2}\right)} \leqslant c, \quad c=\left(n, \alpha, \kappa,\left\|f^{p}\right\|_{L^{1}(Q)}\right) .
$$

\section{3. Неравенства Соболева-Пуанкаре в цилиндре}

Начнем с одного мультипликативного неравенства.

ЛЕмма 3.1. В иилиндре $Q \equiv B_{r}\left(x_{0}\right) \times\left(t_{0}-\tau, t_{0}+\tau\right)$ выполнено неравенство

$$
\begin{aligned}
f_{Q}|u|^{q} d z \leqslant c_{q, n} & \left(f_{Q}|\nabla u|^{q / \gamma} d z\right)^{\gamma n /(n+2)} \\
& \times \sup _{\left|t-t_{0}\right|<\tau}\left(\int_{B_{r}\left(x_{0}\right)}|u|^{2} d x\right)^{q /(n+2)}, \quad \gamma \in\left[1, \frac{n+2}{n}\right],
\end{aligned}
$$

если

$$
\int_{B_{r}\left(x_{0}\right)} u(x, t) d x=0, \quad q \geqslant \frac{n+2}{n} .
$$

ДоказАтельство. Неравенство (см. [3; с. 79-80])

$$
\int_{B_{r}}|u|^{q} d x \leqslant c_{q, n}\left(\int_{B_{r}}|u|^{2} d x\right)^{q /(n+2)} \int_{B_{r}}|\nabla u|^{q n /(n+2)} d x, \quad q \geqslant \frac{n+2}{n},
$$


следует проинтегрировать по $t \in\left(t_{0}-\tau, t_{0}+\tau\right)$ :

$$
f_{Q}|u|^{q} d z \leqslant C(q, n) f_{Q}|\nabla u|^{q n /(n+2)} d z \sup _{\left|t-t_{0}\right|<\tau}\left(\int_{B_{r}}|u|^{2} d x\right)^{q /(n+2)}
$$

и далее применить неравенство Гёльдера к одному из интегралов:

$$
f_{Q}|\nabla u|^{q n /(n+2)} d z \leqslant\left(f_{Q}|\nabla u|^{q / \gamma} d z\right)^{\gamma n /(n+2)}, \quad \gamma \in\left[1, \frac{n+2}{n}\right] .
$$

Лемма доказана.

Теперь приведем неравенство Пуанкаре-Соболева в цилиндре

$$
Q_{r}=Q_{r}\left(z_{0}\right)=B_{r}\left(x_{0}\right) \times\left(t_{0}-\theta r^{2}, t_{0}+\theta r^{2}\right), \quad \theta \in(0,1), \quad Q_{r}\left(z_{0}\right) \in Q_{T},
$$

предполагая, что показатель $p$ удовлетворяет условиям из раздела 1.

Лемма 3.2. Пусть $\nabla v \in L^{p(\cdot)}(Q), Q_{r}\left(z_{0}\right)-$ иилиндр вида (3.2), $p_{2}=\max _{Q_{r}} p$. Тогда найдутся не зависящие от $z_{0} \in Q_{T}$ константы $C$ и $r_{0}$ такие, что

$$
f_{Q_{r}\left(z_{0}\right)}\left|\frac{v}{r}\right|^{p_{2}} d z \leqslant C\left(f_{Q_{r}\left(z_{0}\right)}|\nabla v|^{p(z)} d z+1\right), \quad r \leqslant r_{0},
$$

если

$$
\int_{B_{r}\left(x_{0}\right)} v(x, t) d x=0 \quad \text { npu } \quad t \in\left(t_{0}-\theta r^{2}, t_{0}+\theta r^{2}\right) .
$$

ДокАЗАТЕЛЬСтво. Напишем стандартное неравенство Соболева-Пуанкаре в шаpe $B_{r}=B_{r}\left(x_{0}\right)$ :

$$
\begin{aligned}
& f_{B_{r}}\left|\frac{v}{r}\right|^{s \gamma} d x \leqslant c_{n, s}\left(f_{B_{r}}|\nabla v|^{s} d x\right)^{\gamma} \\
& \text { для всех } s \geqslant 1, \gamma \in\left[1, \frac{n}{n-1}\right], \quad \text { если } \int_{B_{r}} v d x=0 .
\end{aligned}
$$

Отсюда, если $p_{2} / \gamma=p_{1} / q$, где $q \in[1,(n+1) / n]$ и $p_{1}=\min _{Q_{r}} p$, получаем

$$
\begin{aligned}
f_{B_{r}}\left|\frac{v}{r}\right|^{p_{2}} d x & \leqslant c\left(f_{B_{r}}|\nabla v|^{p_{2} / \gamma} d x\right)^{\gamma}=c\left(f_{B_{r}}|\nabla v|^{p_{1} / q} d x\right)^{q p_{2} / p_{1}} \\
& \leqslant c\left(f_{B_{r}}|\nabla v|^{p / q} d x+1\right)^{q+\omega(r) q} \leqslant C\left(f_{B_{r}}|\nabla v|^{p}+1\right) .
\end{aligned}
$$

Здесь, обозначив $\omega(r)=p_{2}-p_{1}$, мы использовали соотношения

$$
\frac{p_{2}}{p_{1}}=\frac{p_{1}+\omega(r)}{p_{1}} \leqslant 1+\omega(r),
$$

а также вытекающее из логарифмического условия (1.3) свойство ограниченности $r^{-\omega(r)} \leqslant C$ с константой, не зависящей от $r \leqslant r_{0}$ и $x_{0}$. Для законного применения (3.4) осталось проверить выполнение условия $\gamma \leqslant n /(n-1)$ при достаточно 
малом $r$. В самом деле, для $r \leqslant r_{0}$, если $r_{0}$ взято таким, что $\omega\left(r_{0}\right) \leqslant 1 /\left(n^{2}-1\right)$, имеем

$$
\gamma=\frac{p_{2}}{p_{1}} q<(1+\omega(r)) q \leqslant \frac{n^{2}}{n^{2}-1} q \leqslant \frac{n}{n-1},
$$

так как $q \leqslant(n+1) / n$. Из (3.5) интегрированием по $t$ выводим (3.3). Лемма доказана.

В дальнейшем, чтобы обеспечить необходимое для неравенства (3.3) условие ортогональности, нам придется менять весовые средние по шару $B$. В связи с этим полезна

Лемма 3.3. Пусть $\rho_{1}, \rho_{2} \in L^{\infty}(B)$,

$$
\int_{B}\left(\rho_{1}-\rho_{2}\right) d x=0, \quad\left\|\rho_{1}-\rho_{2}\right\|_{L^{\infty}} \leqslant c_{0}|B|^{-1} .
$$

Тогда для средних $u_{i}=\int_{B} u \rho_{i} d x, i=1,2$, верно неравенство

$$
\left|u_{1}-u_{2}\right|^{q} \leqslant c_{0}^{q} f_{B}\left|u-u_{i}\right|^{q} d x, \quad i=1,2, \quad q=\text { const } \geqslant 1 .
$$

ДокАЗАтЕЛЬСтво. Из представления

$$
u_{1}-u_{2}=\int_{B} u\left(\rho_{1}-\rho_{2}\right) d x=\int_{B}\left(u-u_{i}\right)\left(\rho_{1}-\rho_{2}\right) d x
$$

по неравенству Гёльдера выводим искомое соотношение

$$
\left|u_{1}-u_{2}\right|^{q} \leqslant c_{0}^{q}\left(f_{B}\left|u-u_{i}\right| d x\right)^{q} \leqslant c_{0}^{q} f_{B}\left|u-u_{i}\right|^{q} d x .
$$

Лемма доказана.

Одним из простых следствий леммы 3.2 является принцип локализации в пространстве $W=W\left(Q_{T}\right)$ (см. определение пространства в разделе 1$)$ :

$$
u \in W, \quad \varphi \in C_{0}^{\infty}\left(Q_{T}\right) \quad \Longrightarrow \quad \varphi u \in W .
$$

Действительно, справедливо утверждение $|\nabla u|^{p} \in L^{1}\left(Q_{T}\right) \Rightarrow|u|^{p} \in L_{\mathrm{loc}}^{1}\left(Q_{T}\right)$, для вывода которого достаточно заметить, что в силу (3.3) $|u|^{p} \in L^{1}\left(Q_{r}\right)$, где $Q_{r}$ - произвольные принадлежащие $Q_{T}$ цилиндры вида (3.2) с достаточно малым радиусом $r$.

\section{4. Неравенство Каччопполи и его следствия}

В этом разделе, помимо уже принятых, используем следующие обозначения:

$$
\begin{gathered}
\Lambda(s)=\Lambda_{t_{0}}(s)=\left\{t \in \mathbb{R}^{1}:\left|t-t_{0}\right|<s\right\}, \\
Q_{R}=Q_{R}\left(z_{0}\right)=Q_{R, \theta R^{2}}\left(z_{0}\right)=B_{R}\left(x_{0}\right) \times \Lambda_{t_{0}}\left(\theta R^{2}\right), \\
u_{R}=u_{R}(t)=f_{B_{R}} u(x, t) d x, \\
h^{p}=\left|h_{1}\right|^{p /(p-1)}+\left|h_{0}\right|, \quad \text { где } h_{0}, h_{1}-\text { функции из условия }(\mathrm{S}) .
\end{gathered}
$$


4.1. Докажем неравенство Каччопполи для решения системы (1.1).

Лемма 4.1. Пусть и - слабое решение системъ (1.1), $Q_{2 r}\left(z_{0}\right) \Subset Q_{T} u p_{2}=$ $\max _{Q_{2 r}}$. Tогдa

$$
\begin{gathered}
\sup _{\Lambda_{t_{0}}\left(\theta r^{2}\right)} \int_{B_{r}\left(x_{0}\right)}\left|u(x, t)-u_{r}(t)\right|^{2} d x+\int_{Q_{r}\left(z_{0}\right)}|\nabla u|^{p(z)} d z \leqslant C\left(\Psi_{1}+\Psi_{2}+\Psi_{3}+\left|Q_{2 r}\right|\right), \\
\Psi_{1}=\frac{1}{\theta r^{2}} \int_{Q_{2 r}\left(z_{0}\right)}\left|u-u_{2 r}(t)\right|^{2} d z, \quad \Psi_{2}=\int_{Q_{2 r}\left(z_{0}\right)}\left|\frac{u-u_{2 r}}{r}\right|^{p_{2}} d z \\
\Psi_{3}=\int_{Q_{2 r}\left(z_{0}\right)} h^{p} d z
\end{gathered}
$$

где постоянная $C$ зависит от $n, N$ и констант $c_{0}, c_{1}, \beta$ из условия $(\mathrm{S})$.

Доказательство. В тождестве (1.5) возьмем $t_{2}=s \in \Lambda\left(\theta r^{2}\right), t_{1} \notin \operatorname{supp} \xi_{0}$ и пробную функцию

$$
\zeta=\left(u-\widetilde{u}_{2 r}(t)\right) \psi
$$

где

$$
\begin{gathered}
\psi(x, t)=\xi^{p_{2}}(x) \xi_{0}^{2}(t), \quad \widetilde{u}(t)=\widetilde{u}_{2 r}(t)=\left(\int_{B_{2 r}} \xi^{p_{2}}(x) d x\right)^{-1} \int_{B_{2 r}} u(x, t) \xi^{p_{2}}(x) d x, \\
\xi \in C_{0}^{\infty}\left(B_{2 r}\right),\left.\quad \xi\right|_{B_{r}} \equiv 1, \quad r|\nabla \xi|<c, \quad 0 \leqslant \xi \leqslant 1, \\
\xi_{0} \in C_{0}^{\infty}\left(\Lambda\left(4 r^{2} \theta\right)\right),\left.\quad \xi_{0}\right|_{\Lambda\left(\theta r^{2}\right)} \equiv 1, \quad \theta r^{2}\left|\xi_{0}^{\prime}\right|<c, \quad 0 \leqslant \xi_{0} \leqslant 1,
\end{gathered}
$$

что возможно, если $и$ достаточно регулярна. После несложных преобразований выводим равенство

$$
\begin{aligned}
& \frac{1}{2} \int_{B_{2 r}}|u(x, s)-\widetilde{u}(s)|^{2} \psi d x+\int_{t_{1}}^{s} \int_{B_{2 r}} \sum_{i} A_{i} \cdot \nabla u_{i} \psi d x \\
& =-p_{2} \int_{t_{1}}^{s} \int_{B_{2 r}} \sum_{i} A_{i} \cdot\left(u_{i}-\widetilde{u}_{i}\right) \xi^{p_{2}-1} \nabla \xi \xi_{0}^{2} d z+\int_{t_{1}}^{s} \int_{B_{2 r}}|u-\widetilde{u}|^{2} \xi^{p_{2}} \xi_{0} \xi_{0}^{\prime} d z
\end{aligned}
$$

уже не содержащее производной по $t$ функции $u$. В случае недостаточной регулярности решения $u$ используем в пробной функции $\zeta$ вместо $u$ сглаживание $u_{h}$ (см. (1.6)) и, исходя из тождества (1.7), получаем сначала равенство (4.3), в котором $u$ и $A_{i}$ заменены на сглаживания $u_{h}$ и $\left(A_{i}\right)_{h}$. Далее, устремляя $h \rightarrow 0$, на основании теоремы 1.5 и (3.6) приходим к самому равенству (4.3). Этот предельный переход использует свойства пространств Орлича, которые приведены в разделе 7 (см., например, (7.5)).

По свойствам средних $u_{r}, u_{2 r}$ и $\widetilde{u}_{2 r}$ имеем соотношения в каждом сечении $t=\mathrm{const}$

$$
\int_{B_{r}}\left|u-u_{r}\right|^{2} d x \leqslant \int_{B_{r}}\left|u-\widetilde{u}_{2 r}\right|^{2} d x \leqslant \int_{B_{2 r}}\left|u-\widetilde{u}_{2 r}\right|^{2} \xi^{p_{2}} d x \leqslant \int_{B_{2 r}}\left|u-u_{2 r}\right|^{2} d x
$$

которые позволяют далее менять в квадратических интегралах среднее $\widetilde{u}_{2 r}$ на $u_{r}$ или $u_{2 r}$. По неравенству Юнга, используя условие $(\mathrm{S})$ и свойства функции $\xi(x)$, 
получаем в цилиндре $Q_{2 r}$ неравенства

$$
\begin{aligned}
& p_{2} \sum_{i}\left|A_{i}\right| \xi^{p_{2}-1}|\nabla \xi|\left|u_{i}-\widetilde{u}_{i}\right| \leqslant \varepsilon|\nabla u|^{p} \xi^{p_{2}}+C_{\varepsilon}\left(\xi^{p_{2}-p}|\nabla \xi|^{p}|u-\widetilde{u}|^{p}+h^{p}\right) \\
& \leqslant \varepsilon|\nabla u|^{p} \xi^{p_{2}}+C_{\varepsilon}\left|\frac{u-\widetilde{u}}{r}\right|^{p}+C_{\varepsilon} h^{p}, \\
&\left|\frac{u-\widetilde{u}}{r}\right|^{p} \leqslant\left|\frac{u-\widetilde{u}}{r}\right|^{p_{2}}+1, \quad \widetilde{u}=\widetilde{u}_{2 r}, \quad \varepsilon>0 .
\end{aligned}
$$

Из (4.3)-(4.5) с учетом условия $(\mathrm{S})$, свойств функций $\xi(x), \xi_{0}(t)$ и леммы 3.3 выводим при достаточно малых $\varepsilon$

$$
\int_{B_{r}}\left|u-u_{r}(t)\right|^{2} d x+\int_{Q_{r}}|\nabla u|^{p} d z \leqslant C\left(\Psi_{1}+\Psi_{2}+\Psi_{3}+\left|Q_{2 r}\right|\right), \quad t \in \Lambda\left(\theta r^{2}\right) .
$$

Лемма доказана.

В лемме 4.1 на геометрию цилиндра $Q_{r, \theta r^{2}}\left(z_{0}\right)$ (т.е. на параметры $r$ и $\theta$ ) никаких ограничений не накладывалось. В следующей лемме рассматривается цилиндр со свойствами (4.6), (4.7), которые можно обеспечить специальным выбором геометрических параметров (см. лемму 2.2 применительно к $f^{p}=|\nabla u|^{p}+h^{p}$, более подробно об этом в разделе 6$)$.

Лемма 4.2. Пусть $и$-слабое решение системы $(1.1), Q_{4 r}=Q_{4 r, \theta(4 r)^{2}}\left(z_{0}\right) \Subset Q_{T}$, $\lambda>0, p_{0}=p\left(z_{0}\right)$ и для некоторой постоянной $c_{3}>1$

$$
c_{3}^{-1} \lambda^{p_{0}} \leqslant f_{Q_{4 r, \theta(4 r)^{2}\left(z_{0}\right)}}\left(|\nabla u|^{p}+h^{p}\right) d z \leqslant c_{3} f_{Q_{r, \theta r^{2}}\left(z_{0}\right)}\left(|\nabla u|^{p}+h^{p}\right) d z \leqslant c_{3}^{2} \lambda^{p_{0}},
$$

¿əe

$$
\theta=\lambda^{2-p_{0}} \text {. }
$$

Тогда, если $\lambda$ достаточно велико, т.е. $\lambda>\lambda_{*} \geqslant 1$, то

$$
X \equiv \sup _{\Lambda_{t_{0}}\left(\theta 4 r^{2}\right)} f_{B_{2 r}\left(x_{0}\right)}\left|\frac{u(x, t)-u_{2 r}(t)}{r}\right|^{2} d x \leqslant c_{4} \lambda^{2},
$$

где постоянные $c_{4}, \lambda_{*}$ зависят от размерностей $n, N$ и констант $c_{0}, c_{1}, \beta$ из усло-

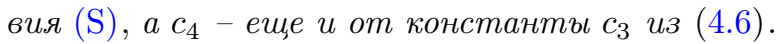

ДокАЗАТЕЛЬСтво. Случаи $p_{0} \geqslant 2$ и $p_{0}<2$ изучаются по-разному. Далее используем обозначения

$$
p_{1}=\min _{Q_{4 r}} p, \quad p_{2}=\max _{Q_{4 r}} p .
$$

Случай $p_{0} \geqslant 2$. Из соотношений (4.2), записанных для цилиндров $Q_{2 r}$ и $Q_{4 r}$, имеем

$$
X \leqslant C\left(\Psi_{1}+\Psi_{2}+\Psi_{3}+\left|Q_{4 r}\right|\right) r^{-(n+2)} .
$$

Здесь слагаемое $\Psi_{1}$ оказывается подчиненным $\Psi_{2}$. В самом деле, по неравенству Юнга

$$
\frac{1}{\theta}\left|\frac{u-u_{4 r}}{r}\right|^{2} \leqslant\left|\frac{u-u_{4 r}}{r}\right|^{p_{2}}+\theta^{-p_{2} /\left(p_{2}-2\right)} .
$$


При этом мы считаем $p_{2}>2$ (случай $p_{2}=2$ далее разобран отдельно). Тогда (см. (4.7)) $\theta^{-p_{2} /\left(p_{2}-2\right)}=\lambda^{p_{2}\left(p_{0}-2\right) /\left(p_{2}-2\right)} \leqslant \lambda^{p_{0}}$ и, интегрируя (4.9) по цилиндру $Q_{4 r}$, получаем

$$
\Psi_{1} \leqslant \Psi_{2}+\lambda^{p_{0}}\left|Q_{4 r}\right| \leqslant \Psi_{2}+c(n) \lambda^{2} r^{n+2} .
$$

Теперь изучим слагаемые $\Psi_{2}$ и $\Psi_{3}$. В соответствии с неравенством (3.3) для функции $u-u_{4 r}$ в цилиндре $Q_{4 r}$ имеем оценку

$$
f_{Q_{4 r}}\left|\frac{u-u_{4 r}}{r}\right|^{p_{2}} d z \leqslant c\left(f_{Q_{4 r}}|\nabla u|^{p(z)} d z+1\right) .
$$

Отсюда в силу (4.6), (4.7)

$$
r^{-(n+2)}\left(\Psi_{2}+\Psi_{3}\right)=C \theta\left(f_{Q_{4 r}}\left(|\nabla u|^{p}+h^{p}\right) d z+1\right) \leqslant C_{1} \lambda^{2-p_{0}} \lambda^{p_{0}}=C_{1} \lambda^{2} .
$$

Из полученных оценок при достаточно большом $\lambda$ следует неравенство (4.8).

Равенство $p_{2}=2$ возможно только, если $p_{0}=2$. Но тогда в наших оценках $\Psi_{1}=\Psi_{2}$, так как $\theta=\lambda^{2-p_{0}}=1$. Осталось заметить, что прежняя оценка для $\Psi_{2}$ проходит при $p_{2}=2$.

Случай $p_{0}<2$. В тождестве (1.5) полагаем (с теми же оговорками насчет использования сглаживаний, что при выводе леммы 4.1)

$$
\zeta=(u-\widetilde{u}(t)) \xi^{2}(x),
$$

где

$$
\begin{aligned}
& \xi \in C_{0}^{\infty}\left(B_{4 r}\right),\left.\quad \xi\right|_{B_{2 r}} \equiv 1, \quad 0 \leqslant \xi \leqslant 1, \quad r|\nabla \xi|<2, \\
& \widetilde{u}(t)=\widetilde{u}_{4 r}(t)=\left(\int_{B_{4 r}} \xi^{2} d x\right)^{-1} \int_{B_{4 r}} u(x, t) \xi^{2}(x) d x,
\end{aligned}
$$

считая $t_{2}=s \in \Lambda_{t_{0}}\left(4 r^{2} \theta\right), t_{1}=\widehat{t} \in \Lambda_{t_{0}}\left(\theta r^{2}\right)$, и более точный выбор $\widehat{t}$ укажем позже. Тогда

$$
\begin{aligned}
& \frac{1}{2} \int_{B_{4 r}}|u(x, s)-\widetilde{u}(s)|^{2} \xi^{2} d x+\int_{\widehat{t}}^{s} \int_{B_{4 r}} \sum_{i} A_{i} \cdot \nabla u_{i} \xi^{2} d z \\
& \quad=\frac{1}{2} \int_{B_{4 r}}|u(x, \widehat{t})-\widetilde{u}(\widehat{t})|^{2} \xi^{2} d x+2 \int_{\widehat{t}}^{s} \int_{B_{4 r}} \sum_{i} A_{i} \cdot\left(u_{i}-\widetilde{u}_{i}\right) \xi \nabla \xi d z
\end{aligned}
$$

Оценим последний интеграл $I(s)$. По неравенству Юнга и условию $(\mathrm{S})$, см. также (4.1), имеем

$$
\sum_{i}\left|A_{i}\right|\left|u_{i}-\widetilde{u}_{i}\right| \xi|\nabla \xi| \leqslant \varepsilon \frac{1}{\theta r^{2}}|u-\widetilde{u}|^{2} \xi^{2}+C_{\varepsilon} \theta\left(|\nabla u|^{2\left(p_{2}-1\right)}+h^{2\left(p_{2}-1\right)}+1\right), \quad \varepsilon>0
$$

и, соответственно, $I(s) \leqslant \varepsilon I_{1}(s)+C_{\varepsilon} I_{2}(s)$, где при достаточно малом $\varepsilon$ слагаемое

$$
\varepsilon I_{1}(s) \equiv \varepsilon \frac{1}{\theta r^{2}} \int_{\widehat{t}}^{s} \int_{B_{4 r}}|u-\widetilde{u}|^{2} \xi^{2} d z, \quad s \in \Lambda_{t_{0}}\left(4 r^{2} \theta\right),
$$


впоследствии поглощается левой частью неравенства (4.11). Отсюда, учитывая условие $(\mathrm{S})$ и свойства средних $u_{2 r}, u_{4 r}, \widetilde{u}=\widetilde{u}_{4 r}$, подобные свойствам (4.4), выводим из (4.10)

$$
\begin{aligned}
X & \leqslant \sup _{\Lambda_{t_{0}}\left(4 r^{2} \theta\right)} f_{B_{4 r}}\left|\frac{u-\widetilde{u}}{r}\right|^{2} \xi^{2} d x \\
& \leqslant C f_{B_{4 r}}\left|\frac{u(x, \widehat{t})-u_{4 r}(\widehat{t})}{r}\right|^{2} d x+C f_{Q_{4 r}}\left(\theta^{2}\left(|\nabla u|^{2\left(p_{2}-1\right)}+h^{2\left(p_{2}-1\right)}+1\right)+\theta h^{p}\right) d z \\
& \equiv X_{1}+X_{2} .
\end{aligned}
$$

По неравенству Соболева $\left(2 n /(n+2)<p_{1}\right)$ имеем

$$
f_{B_{4 r}}\left|\frac{u(x, \widehat{t})-u_{4 r}(\widehat{t})}{r}\right|^{2} d x \leqslant C_{1}\left(f_{B_{4 r}}|\nabla u(x, \widehat{t})|^{p_{1}} d x\right)^{2 / p_{1}} \leqslant C_{2}\left(f_{Q_{4 r}}|\nabla u|^{p_{1}} d x\right)^{2 / p_{1}}
$$

если $\widehat{t} \in \Lambda_{t_{0}}\left(\theta r^{2}\right)$ выбрано так, что для функции $f \equiv|\nabla u|^{p_{1}}$ выполнены неравенства

$$
\int_{B_{4 r}} f(\widehat{t}, x) d x \leqslant\left|\Lambda_{t_{0}}\left(\theta r^{2}\right)\right|^{-1} \int_{Q_{4 r}} f(z) d z, \quad f_{B_{4 r}} f(\widehat{t}, x) d x \leqslant 16 f_{Q_{4 r}} f(z) d z .
$$

Тогда в силу (4.6) при достаточно больших $\lambda \geqslant \lambda_{*}$

$$
X_{1} \leqslant c\left(f_{Q_{4 r}}|\nabla u|^{p} d z+1\right)^{2 / p_{1}} \leqslant C \lambda^{2 p_{0} / p_{1}} \leqslant C_{1} \lambda^{2} .
$$

K заключительному неравенству здесь приводят те же аргументы, что и в случае (2.11), поскольку

$$
\frac{p_{0}}{p_{1}}=1+\frac{p_{0}-p_{1}}{p_{1}} \leqslant 1+\omega(r), \quad \text { где } \quad \omega(r)=p_{2}-p_{1},
$$

и $\lambda^{\omega(r)} \leqslant C$ в силу $(1.3)$.

Изучая слагаемое $X_{2}$, считаем параметр $\lambda$ столь большим и, значит, радиус $r$ столь малым (см. замечание в разделе 2 ), что $p_{2}<2$ и $2 p_{2}-2<p_{1}$, т.е. $p_{2}-p_{1}<$ $2-p_{2}$. Тогда по неравенству Гёльдера и условию (4.6)

$$
\begin{aligned}
\int_{Q_{4 r}} h^{2\left(p_{2}-1\right)} d z & \leqslant\left(\int_{Q_{4 r}} h^{p_{1}} d z\right)^{2\left(p_{2}-1\right) / p_{1}} \\
& \leqslant\left(f_{Q_{4 r}} h^{p} d z+1\right)^{2\left(p_{2}-1\right) / p_{1}} \leqslant c \lambda^{2\left(p_{2}-1\right) p_{0} / p_{1}},
\end{aligned}
$$

и оценка остается в силе, если $h$ заменить на $|\nabla u|$. Отсюда те же соображения, что и при изучении $X_{1}$, дают неравенство

$$
X_{2} \leqslant C\left(\theta^{2} \lambda^{2\left(p_{2}-1\right) p_{0} / p_{1}}+\theta \lambda^{p_{0}}\right)=C\left(\lambda^{2}+\lambda^{2} \lambda^{2-2 p_{0}+2\left(p_{2}-1\right) p_{0} / p_{1}}\right) \leqslant C_{1} \lambda^{2},
$$

поскольку $\lambda^{\omega(r)} \leqslant C$ и

$$
1-p_{0}+\left(p_{2}-1\right) \frac{p_{0}}{p_{1}} \leqslant \frac{p_{0}}{p_{1}}\left(p_{2}-p_{1}\right) \leqslant 2 \omega(r), \quad \text { где } \quad \omega(r)=p_{2}-p_{1} .
$$

Из (4.11) и оценок для $X_{1}$ и $X_{2}$ вытекает неравенство (4.8). Лемма доказана. 
ЛЕмма 4.3. В условиях леммы 4.2 при достаточно большом $\lambda>\lambda_{*} \geqslant 1$

$$
f_{Q_{r}}|\nabla u|^{p} d z \leqslant c_{5}\left[1+f_{Q_{2 r}} h^{p} d z+\left(f_{Q_{2 r}}|\nabla u|^{2 / \gamma^{\prime}} d z\right)^{p_{0} \gamma^{\prime} / 2}+\left(f_{Q_{2 r}}|\nabla u|^{p_{2} / \gamma} d z\right)^{\gamma}\right]
$$

где $\gamma, \gamma^{\prime} \in[1,(n+2) / n], p_{0}=p\left(z_{0}\right), p_{2}=\max _{Q_{2 r}} p, Q_{r}=Q_{r, \theta r^{2}}\left(z_{0}\right), Q_{2 r}=Q_{r, \theta(2 r)^{2}}\left(z_{0}\right)-$ иилиндры со свойством (4.6), а константа с 5 того же типа, что $c_{4}$ в (4.8).

ДокАзАтЕльство. Полагая $v=|\nabla u|$, в силу (4.2) имеем

$$
\int_{Q_{r}} v^{p} d z \leqslant C\left(\Psi_{1}+\Psi_{2}+\Psi_{3}+\left|Q_{2 r}\right|\right) .
$$

Для оценки $\Psi_{1}$ применим неравенство (3.1) (при $q=2$ и $\gamma^{\prime} \in[1,(n+2) / n]$ ), а затем соотношения (4.7) и (4.8):

$$
\begin{aligned}
\left|Q_{2 r}\right|^{-1} \Psi_{1} & \leqslant C\left(\theta r^{2}\right)^{-1}\left(\sup _{\left|t-t_{0}\right|<4 \theta r^{2}} \int_{B_{2 r}}\left|u-\bar{u}_{2 r}\right|^{2} d x\right)^{2 /(n+2)}\left(f_{Q_{2 r}} v^{2 / \gamma^{\prime}} d z\right)^{\gamma^{\prime} n /(n+2)} \\
& \leqslant C \lambda^{p_{0}-2} r^{-2}\left(\lambda^{2} r^{n+2}\right)^{2 /(n+2)}\left(f_{Q_{2 r}} v^{2 / \gamma^{\prime}} d z\right)^{\gamma^{\prime} n /(n+2)} \\
& =c \lambda^{p_{0}-2 n /(n+2)}\left(f_{Q_{2 r}} v^{2 / \gamma^{\prime}} d z\right)^{\gamma^{\prime} n /(n+2)} \leqslant \varepsilon \lambda^{p_{0}}+C_{\varepsilon}\left(f_{Q_{2 r}} v^{2 / \gamma^{\prime}} d z\right)^{\gamma^{\prime} p_{0} / 2},
\end{aligned}
$$

где в конце использовано неравенство Юнга с $\varepsilon \in(0,1)$.

Аналогично, применяя на этот раз (3.1) при $q=p_{2}$ и $\gamma \in[1,(n+2) / n]$, получаем оценку для $\Psi_{2}$

$$
\begin{aligned}
\left|Q_{2 r}\right|^{-1} \Psi_{2} & \leqslant C r^{-p_{2}}\left(r^{n+2} \lambda^{2}\right)^{p_{2} /(n+2)}\left(f_{Q_{2 r}} v^{p_{2} / \gamma} d z\right)^{\gamma n /(n+2)} \\
& =C \lambda^{2 p_{2} /(n+2)}\left(f_{Q_{2 r}} v^{p_{2} / \gamma} d z\right)^{\gamma n /(n+2)} \leqslant \varepsilon \lambda^{p_{2}}+C_{\varepsilon}\left(f_{Q_{2 r}} v^{p_{2} / \gamma} d z\right)^{\gamma},
\end{aligned}
$$

где, как и при выводе леммы 4.2, можно показать, что $\lambda^{p_{2}}=\lambda^{p_{0}} \cdot \lambda^{p_{2}-p_{0}} \leqslant C \lambda^{p_{0}}$. В итоге из (4.13)-(4.15) и (4.6) следует (4.12), если $\varepsilon$ достаточно мало. Лемма доказана.

4.2. Собранного материала достаточно, чтобы доказать обратное неравенство Гёльдера для градиента решения системы (1.1).

Предварительно введем величину

$$
\varsigma=\frac{1}{m}\left[\alpha\left(\frac{2 n}{n+2}\right)^{-1}-1\right]>0,
$$

где $m \geqslant 2$ столь велико, что $\varsigma<2 / n$. Последнее неравенство обеспечивает необходимое в дальнейшем включение

$$
s=\frac{n+2}{n(1+\varsigma)} \in\left(1, \frac{n+2}{n}\right) .
$$


ЛЕмма 4.4. В условиях леммы 4.3 выполнены неравенства для $v=|\nabla u|$

$$
\begin{array}{ll}
f_{Q_{r}} v^{p} d z \leqslant c_{6}\left(1+\left(f_{Q_{2 r}} v^{2 / s} d z\right)^{p_{0} s / 2}+f_{Q_{2 r}} h^{p} d z\right), & \text { если } \quad p_{0} \leqslant 2, \\
f_{Q_{r}} v^{p} d z \leqslant c_{6}\left(1+\left(f_{Q_{2 r}} v^{p / s} d z\right)^{s}+f_{Q_{2 r}} h^{p} d z\right), & \text { еслu } \quad p_{0}>2,
\end{array}
$$

где s определено в (4.17), (4.16) и с 6 - константа того же типа, что $c_{4}$ в (4.8).

ДоказАтеЛЬСтво. $1^{\circ}$. Пусть $p_{0} \leqslant 2$. Положим $p_{1}=\min _{Q_{2 r}} p, p_{2}=\max _{Q_{2 r}} p$, $\omega(r)=p_{2}-p_{1}$. В оценке (4.12) берем $\gamma^{\prime}=s$ и $\gamma=s p_{2} / p_{1}$. Это законно, так как $\gamma^{\prime}, \gamma \in(1,(n+2) / n]$, см. (4.17) и следующие неравенства:

$$
1<\gamma=\frac{p_{2}}{p_{1}} s \leqslant\left(1+p_{2}-p_{1}\right) s \leqslant(1+\varsigma) \frac{n+2}{n(1+\varsigma)}=\frac{n+2}{n},
$$

которые верны, если $r$ столь мало, что $\omega(r) \leqslant \varsigma$. Тогда неравенство $(4.12)$ принимает вид

$$
f_{Q_{r}} v^{p} d z \leqslant c_{4}\left[1+f_{Q_{2 r}} h^{p} d z+\left(f_{Q_{2 r}} v^{2 / s} d z\right)^{p_{0} s / 2}+\left(f_{Q_{2 r}} v^{p_{1} / s} d z\right)^{\gamma}\right]
$$

и последний интеграл подлежит оценке. Поскольку $\gamma=s+s \omega(r)($ см. (4.19)), $s>1$ и $p_{1} \leqslant 2$, нетрудно вывести, что

$$
\begin{aligned}
\left(f_{Q_{2 r}} v^{p_{1} / s} d z\right)^{\gamma} & \leqslant\left(f_{Q_{2 r}} v^{p_{1} / s} d z\right)^{s}\left(f_{Q_{2 r}} v^{p_{1}} d z\right)^{\omega(r)} \\
& \leqslant\left(f_{Q_{2 r}} v^{2 / s} d z\right)^{p_{1} s / 2}\left[\left|Q_{2 r}\right|^{-\omega(r)}\left(\int_{Q_{2 r}} v^{p} d z+1\right)\right] .
\end{aligned}
$$

Заметим, что $\left|Q_{2 r}\right|=2 \omega_{n}\left(\theta r^{2}\right) r^{n}$ и величины $r, \theta r^{2}$ имеют оценку типа (2.10). Поэтому выражение в квадратной скобке ограничено допустимой константой в силу логарифмического условия на показатель $p(z)$. Отсюда и из (4.20) следует $(4.18)_{1}$, так как $p_{1} \leqslant p_{0}$.

$2^{\circ}$. Пусть $p_{0}>2$; тогда, тем более, $p_{2}>2$. В неравенстве $(4.12)$ возьмем такие $\gamma$ и $\gamma^{\prime}$, что

$$
\frac{p_{2}}{p_{1}} s=\gamma \leqslant \gamma^{\prime}<\frac{n+2}{n}
$$

(условие $\gamma=s p_{2} / p_{1}<(n+2) / n$ проверено ранее). Тогда ввиду очевидных неравенств $2 / \gamma^{\prime} \leqslant 2 / \gamma<p_{2} / \gamma$ в (4.12) последний интеграл доминирует над предшествующим, при этом имеет место оценка (аналогичная (4.21))

$$
\begin{aligned}
\left(f_{Q_{2 r}} v^{p_{2} / \gamma} d z\right)^{\gamma} & =\left(f_{Q_{2 r}} v^{p_{1} / s} d z\right)^{s p_{2} / p_{1}} \\
& \leqslant C\left(f_{Q_{2 r}} v^{p_{1} / s} d z\right)^{s} \leqslant C\left(1+f_{Q_{2 r}} v^{p / s} d z\right)^{s}
\end{aligned}
$$

которая и приводит к неравенству $(4.18)_{2}$. Лемма доказана. 
Неравенства (4.18) можно объединить в одно:

$$
\begin{gathered}
f_{Q_{r}}|\nabla u|^{p(z)} d z \leqslant c_{6}\left(1+\left(f_{Q_{2 r}}|\nabla u|^{p(z) / q(z)} d z\right)^{q_{0}}+f_{Q_{2 r}} h^{p(z)} d z\right), \quad q_{0}=q\left(z_{0}\right), \\
q(z)= \begin{cases}s, & \text { если } p(z) \geqslant 2, \\
\frac{p(z) s}{2}, & \text { если } p(z)<2 .\end{cases}
\end{gathered}
$$

Показатель $q(z)$ наследует от $p(z)$ логарифмический модуль непрерывности.

\section{5. Вариант леммы Геринга}

Пусть $Q=O \times\left(t_{1}, t_{2}\right) \subset \mathbb{R}_{z}^{n+1}, z=(x, t), O$ - область в $\mathbb{R}^{n}$, функция $q(z)$ такова, что

$$
\begin{gathered}
1<\gamma_{1} \leqslant q(z) \leqslant \gamma_{2}<\infty, \quad z \in \bar{Q} \\
\left|q(z)-q\left(z^{\prime}\right)\right| \leqslant \omega\left(\left|z-z^{\prime}\right|\right), \quad z, z^{\prime} \in \bar{Q}, \quad\left|z-z^{\prime}\right|<\frac{1}{2}, \quad \omega(s)=\frac{\kappa}{\ln (1 / s)}, \quad \kappa>0
\end{gathered}
$$

и аналогичным требованиям подчинена функция $p(z)$.

Пусть $z_{0}=\left(x_{0}, t_{0}\right) \in Q, q\left(z_{0}\right)=q_{0}, p\left(z_{0}\right)=p_{0}$ и $Q_{r, \tau}\left(z_{0}\right)=B_{r}\left(x_{0}\right) \times\left(t_{0}-\tau, t_{0}+\tau\right)-$ параболические цилиндры с центром в точке $z_{0}$. Следующее условное утверждение является модификацией леммы Геринга и обслуживает параболические уравнения переменного порядка нелинейности.

Теорема 5.1. Пусть функиии $F \geqslant 0, G \geqslant 0$ таковы, что $F^{p}, G^{p} \in L^{1}(Q), u$ пусть для некоторых констант $\lambda_{0} \geqslant 1, c_{0} \geqslant 1, c_{1} \geqslant 1, l>0$ и для п.в. точек

$$
z_{0} \in E(\lambda)=\{z \in Q: F>\lambda\}, \quad \lambda \geqslant \lambda_{0},
$$

имеются $r=r\left(z_{0}, \lambda\right)>0, \tau=\tau\left(z_{0}, \lambda\right)>0$ и соответствующие этим параметрам конщентрические иилиндрь

$$
Q^{i}=Q_{2^{i} r, 2^{2 i} \tau}\left(z_{0}\right) \subset Q, \quad i=1,3,
$$

такие, что

$$
\begin{gathered}
r \leqslant c_{1} \lambda^{-l}, \quad \tau \leqslant c_{1} \lambda^{-l}, \\
c_{0}^{-1} f_{Q^{3}} F^{p} d z \leqslant \lambda^{p_{0}} \leqslant c_{0}\left(\left(f_{Q^{1}} F^{p / q} d z\right)^{q_{0}}+f_{Q^{1}} G^{p} d z\right) .
\end{gathered}
$$

Тогда найдется такое $\delta_{0}>0$, что

$$
\int_{Q} F^{p+\delta} d z \leqslant C \int_{Q}\left(F^{p}+G^{p+\delta}+1\right) d z, \quad \delta \in\left(0, \delta_{0}\right) .
$$

Постоянные $\delta_{0}$ и $C$ зависят от размерности $n$ и констант $\gamma_{1}, \gamma_{2}, \kappa, l, c_{0}, c_{1}$ из (5.1)-(5.4), а постоянная $C$ - еще и от $\lambda_{0}$. 
ДокАЗАТЕЛЬСтво. $1^{\circ}$. Оценим интегралы по $Q^{1}$ из (5.4). Полагаем

$$
q_{1}=\min _{Q^{1}} q, \quad q_{2}=\max _{Q^{1}} q, \quad p_{1}=\min _{Q^{1}} p, \quad p_{2}=\max _{Q^{1}} p .
$$

В силу (5.1), (5.3)

$$
\lambda^{q_{2}-q_{1}} \leqslant C, \quad \lambda^{p_{2}-p_{1}} \leqslant C,
$$

где $C$ не зависит от $z_{0}, \lambda$ и $r$. Применяя неравенство Гёльдера и (5.4), получаем

$$
\begin{aligned}
I_{1} \equiv\left(f_{Q^{1}} F^{p / q} d z\right)^{q_{0}} & \leqslant\left(f_{Q^{1}} F^{q_{1} p / q} d z\right)^{\left(q_{0}-1\right) / q_{1}} \cdot f_{Q^{1}} F^{p / q} d z \\
& \leqslant\left(f_{Q^{1}} F^{p} d z+1\right)^{\left(q_{0}-1\right) / q_{1}} \cdot f_{Q^{1}} F^{p / q} d z \\
& \leqslant c_{1} \lambda^{p_{0}\left(q_{0}-1\right) / q_{1}} f_{Q^{1}} F^{p / q} d z
\end{aligned}
$$

с учетом того, что

$$
f_{Q^{1}} F^{p} d z \leqslant \frac{\left|Q^{3}\right|}{\left|Q^{1}\right|} f_{Q^{3}} F^{p} d z
$$

Поскольку

$$
f_{Q^{1}} F^{p / q} d z \leqslant(\varepsilon \lambda)^{p_{2} / q_{1}}+\frac{1}{\left|Q^{1}\right|} \int_{Q^{1} \cap E(\varepsilon \lambda)} F^{p / q} d z, \quad \varepsilon \in(0,1),
$$

имеем в силу (5.6)

$$
I_{1} \leqslant c_{2}\left[\lambda^{p_{0}} \varepsilon^{\gamma_{1} / \gamma_{2}}+\lambda^{p_{0}\left(q_{0}-1\right) / q_{0}} \frac{1}{\left|Q^{1}\right|} \int_{Q^{1} \cap E(\varepsilon \lambda)} F^{p / q} d z\right] .
$$

Полагая $D(\lambda)=\{z \in Q: G(z)>\lambda\}$, снова в силу (5.6) устанавливаем

$$
I_{2} \equiv f_{Q^{1}} G^{p} d z \leqslant c_{3}\left[\lambda^{p_{0}} \varepsilon^{\gamma_{1}}+\frac{1}{\left|Q^{1}\right|} \int_{Q^{1} \cap D(\varepsilon \lambda)} G^{p} d z\right], \quad \varepsilon \in(0,1) .
$$

Из (5.4) и оценок для $I_{1}, I_{2}$, выбирая $\varepsilon$ достаточно малым, выводим

$$
\int_{Q^{3}} F^{p} d z \leqslant c\left[\lambda^{p_{0}\left(q_{0}-1\right) / q_{0}} \int_{Q^{1} \cap E(\varepsilon \lambda)} F^{p / q} d z+\int_{Q^{1} \cap D(\varepsilon \lambda)} G^{p} d z\right] .
$$

Согласно (5.6) эта оценка сохраняет свой вид, если значения $q_{0}, p_{0}$ заменить значениями $\widehat{q}, \widehat{p}$, которые даются теоремой о среднем значении

$$
\int_{Q^{1} \cap E(\varepsilon \lambda)} \lambda^{p(q-1) / q} F^{p / q} d z=\lambda^{\widehat{p}(\widehat{q}-1) / \widehat{q}} \int_{Q^{1} \cap E(\varepsilon \lambda)} F^{p / q} d z .
$$

Поэтому оценка (5.7) принимает вид

$$
\int_{Q^{3}} F^{p} d z \leqslant c\left[\int_{Q^{1} \cap E(\varepsilon \lambda)} \lambda^{p(q-1) / q} F^{p / q} d z+\int_{Q^{1} \cap D(\varepsilon \lambda)} G^{p} d z\right] .
$$


$2^{\circ}$. Итак, для п.в. точек $z_{0} \in E(\lambda), \lambda \geqslant \lambda_{0}$, существуют цилиндры $Q^{1}, Q^{3}$ вида (5.2) с центром в точке $z_{0}$, для которых выполнена оценка (5.8). По теореме Витали найдется счетное семейство попарно непересекающихся цилиндров $\left\{Q_{j}^{1}\right\}$ такое, что $E(\lambda) \subset \cup_{j} Q_{j}^{3}$, и из (5.8) получим

$$
\int_{E(\lambda)} F^{p} d z \leqslant c\left[\int_{E(\lambda \varepsilon)} \lambda^{p(q-1) / q} F^{p / q} d z+\int_{D(\lambda \varepsilon)} G^{p}(z) d z\right] .
$$

В случае постоянных $q>1$ и $\delta>0$ верна формула интегрирования

$$
\begin{aligned}
I & \equiv \int_{E\left(\lambda_{0}\right)} F^{p+\delta} d z=-\int_{\lambda_{0}}^{\infty} \lambda^{\delta} d_{\lambda} \int_{E(\lambda)} F^{p} d z \\
& =\delta \int_{\lambda_{0}}^{\infty} \lambda^{\delta-1} \int_{E(\lambda)} F^{p} d z d \lambda+\lambda_{0}^{\delta} \int_{E\left(\lambda_{0}\right)} F^{p} d z
\end{aligned}
$$

которая справедлива и для переменных показателей, в чем можно убедиться, рассматривая кусочно-постоянные приближения. Поэтому из (5.10) и (5.9) выводим

$$
\begin{aligned}
I \leqslant c_{1} \delta & {\left[\int_{s_{0}}^{\infty} \lambda^{\delta-1} \int_{E(\lambda)} \lambda^{p(q-1) / q} F^{p / q} d z d \lambda+\int_{s_{0}}^{\infty} \lambda^{\delta-1} \int_{D(\lambda)} G^{p} d z d \lambda\right] } \\
& +\lambda_{0}^{\delta} \int_{E\left(\lambda_{0}\right)} F^{p} d z, \quad s_{0}=\varepsilon \lambda_{0} .
\end{aligned}
$$

Оценим сверху слагаемые в квадратных скобках. Очевидно, что (см. формулу типа (5.10) для $G$ вместо $F)$

$$
\delta \int_{s_{0}}^{\infty} \lambda^{\delta-1} \int_{D(\lambda)} G^{p} d z d \lambda \leqslant \int_{D\left(s_{0}\right)} G^{p+\delta} d z .
$$

Кроме того, из равенства типа (5.10)

$$
\begin{aligned}
I_{0} & \equiv \int_{E\left(s_{0}\right)} F^{p+\delta} d z \\
& =\int_{s_{0}}^{\infty}(\delta+1) \lambda^{\delta} \int_{E(\lambda)} \lambda^{p(q-1) / q-1} F^{p / q} d z d \lambda+s_{0}^{\delta} \int_{E\left(s_{0}\right)} s_{0}^{p(q-1) / q} F^{p / q} d z,
\end{aligned}
$$

следует оценка

$$
\int_{s_{0}}^{\infty} \lambda^{\delta-1} \int_{E(\lambda)} \lambda^{p(q-1) / q} F^{p / q} d z d \lambda \leqslant \frac{1}{\delta+1} I_{0} \leqslant \frac{1}{\delta+1}\left[I+\lambda_{0}^{\gamma_{2}+\delta}|Q|\right] .
$$

В результате из (5.11) получаем

$$
I \leqslant \frac{c_{1} \delta}{\delta+1}\left(I+\lambda_{0}^{\gamma_{2}+\delta}|Q|\right)+\lambda_{0}^{\delta} \int_{E\left(\lambda_{0}\right)} F^{p} d z+c_{1} \int_{D\left(s_{0}\right)} G^{p+\delta} d z .
$$

Взяв $2 \delta_{0}=1 / c_{1}$, приходим к оценкам с независящими от $\delta \in\left(0, \delta_{0}\right)$ константами

$$
\begin{aligned}
& \int_{E\left(\lambda_{0}\right)} F^{p+\delta} d z \leqslant c \int_{Q}\left(F^{p}+G^{p+\delta}+1\right) d z, \\
& \int_{Q} F^{p+\delta} d z \leqslant C \int_{Q}\left(F^{p}+G^{p+\delta}+1\right) d z, \quad \delta \in\left(0, \delta_{0}\right),
\end{aligned}
$$

из которых следует утверждение теоремы 5.1. 


\section{6. Локальная повышенная суммируемость}

Перейдем к непосредственному выводу неравенства (1.4), для чего суммируем полученные в разделе 4 результаты в такой форме, чтобы воспользоваться теоремой 5.1. Дальнейшие построения проводятся по отношению к некоторому фиксированному цилиндру $Q \Subset Q_{T}$. Устраиваем локализацию в окрестности любой точки $z_{0} \in Q$ с помощью специально построенных в разделе 2 цилиндров, учитывая несколько факторов: значение $p_{0}=p\left(z_{0}\right)$, расстояние до границы $\partial Q$, значения $|\nabla u|$ и функций $h_{i}$ из условия $(\mathrm{S})$ в точке $z_{0}$. Отметим особо различие в геометрии цилиндров для случаев $p_{0}<2$ и $p_{0} \geqslant 2$.

ДоКАЗАТЕЛЬСТво тЕОРЕМЫ 1.1. Выделим несколько шагов.

$1^{\circ}$. Применяя лемму 2.2 к функции $f^{p}=|\nabla u|^{p}+h^{p}$, убеждаемся в выполнении условий (4.6), (4.7), если в них большой параметр $\lambda$ заменить на $\widetilde{\lambda}=\lambda / \rho\left(z_{0}\right)$, где вес $\rho(z)$ определен в (2.6). Это относится к тем точкам $z_{0}$, в которых $f\left(z_{0}\right)>\widetilde{c} \lambda / \rho\left(z_{0}\right)$, $\lambda>\lambda_{0} \geqslant 1$. Константы $\widetilde{c}$ и $\lambda_{0}$ могут быть точно указаны, см. раздел 2, а константа $c_{3}$ из (4.6) зависит лишь от размерности $n$. По лемме 2.2 получаем также неравенство

$$
c^{-1} f_{Q^{3}}\left(|\nabla u|^{p}+h^{p}\right) d z \leqslant \widetilde{\lambda}^{p_{0}} \leqslant c f_{Q^{0}}\left(|\nabla u|^{p}+h^{p}\right) d z, \quad c>1 .
$$

$2^{\circ}$. Коль скоро обеспечены условия (4.6) и (4.7), можно считать доказанным неравенство (4.22). Совмещая различные обозначения из разделов 2 и 4 для одних и тех же цилиндров $Q_{r}=Q^{0}, Q_{2 r}=Q^{1}, Q_{8 r}=Q^{3}$, записываем (4.22) в следующем виде:

$$
f_{Q^{0}}|\nabla u|^{p} d z \leqslant c_{6}\left(1+\left(f_{Q^{1}}|\nabla u|^{p / q} d z\right)^{q_{0}}+f_{Q^{1}} h^{p} d z\right), \quad q_{0}=q\left(z_{0}\right),
$$

что вместе с (6.1) дает для функции $f^{p}=|\nabla u|^{p}+h^{p}$ неравенство

$$
C^{-1} f_{Q^{3}} f^{p} d z \leqslant \tilde{\lambda}^{p_{0}} \leqslant C\left(1+\left(f_{Q^{1}} f^{p / q} d z\right)^{q_{0}}+f_{Q^{1}} h^{p} d z\right), \quad C>1 .
$$

Из соотношений $1 / 2 \leqslant d(z) / d\left(z_{0}\right) \leqslant 3 / 2, z \in Q^{3}$, следуют аналогичные соотношения для веса $\rho(z)$ :

$$
0<\widehat{c} \leqslant \frac{\rho(z)}{\rho\left(z_{0}\right)} \leqslant \widehat{c}^{-1}, \quad z \in Q^{3} .
$$

Тогда из (6.2), полагая

$$
F^{p}=\left(f \rho \widetilde{c}^{-1}\right)^{p}, \quad G^{p}=\left(h^{p}+1\right) \rho^{p},
$$

имеем $F\left(z_{0}\right)>\lambda$, при этом

$$
\widehat{c}_{0}^{-1} f_{Q^{3}} F^{p} d z \leqslant \lambda^{p_{0}} \leqslant \widehat{c}_{0}\left(\left(f_{Q^{1}} F^{p / q} d z\right)^{q_{0}}+f_{Q^{1}} G^{p} d z\right), \quad \widehat{c}_{0}>1 .
$$

Таким образом, для функции $F$ из (6.3) проверено условие (5.4), причем показатели $p$ и $q$, с которыми мы работаем, подчинены соотношениям (5.1). 
$3^{\circ}$. Геометрические параметры построенных в лемме 2.1 цилиндров (см. (2.8)), удовлетворяют условиям (2.10):

$$
\begin{gathered}
r \leqslant c \widetilde{\lambda}^{-2 /(n+2)}=c \lambda^{-2 /(n+2)} \rho_{0}^{2 /(n+2)} \leqslant c_{1} \lambda^{-l}, \\
\tau=\theta r^{2}=\widetilde{\lambda}^{2-p_{0}} r^{2} \leqslant c^{2} \widetilde{\lambda}^{2 n /(n+2)-\alpha}=c^{2} \lambda^{2 n /(n+2)-\alpha} \rho_{0}^{\alpha-2 n /(n+2)} \leqslant c_{1} \lambda^{-l}
\end{gathered}
$$

для подходящих констант $c_{1}, l>0$. Таким образом, проверены неравенства (5.3). В итоге все предположения теоремы 5.1 выполнены для функции $F$ из (6.3).

$4^{\circ}$. По теореме 5.1 при достаточно малом $\delta>0$ конечна норма $\left\|F^{q+\delta}\right\|_{L^{1}(Q)}$, если $G^{q+\delta} \in L^{1}(Q)$. Возвращаясь от функций $F$ и $G$ к исходным функциям $|\nabla u|$ и $h$, выводим отсюда свойство локальной повышенной суммируемости (1.4). Теорема 1.1 доказана.

\section{7. О пространствах Соболева-Орлича}

7.1. Пусть $p$ - измеримая в $Q_{T}$ функция такая, что $1<\alpha \leqslant p(z) \leqslant \beta<\infty$. Через $L^{p(\cdot)}\left(Q_{T}\right)$ обозначим множество всех измеримых в $Q_{T}$ функций $f(z)$ таких, что

$$
A_{p}(f)=\int_{Q_{T}}|f(z)|^{p(z)} d z<\infty .
$$

Класс $L^{p(\cdot)}\left(Q_{T}\right)$ будет рефлексивным банаховым пространством, называемым пространством Орлича, если норму $\|\cdot\|_{L^{p(\cdot)}\left(Q_{T}\right)}=\|\cdot\|_{L^{p(\cdot)}}$ определить равенством

$$
\|f\|_{L^{p(\cdot)}}=\inf \left\{\lambda>0: A_{p}\left(\lambda^{-1} f\right) \leqslant 1\right\} .
$$

Заметим, что нижняя грань в (7.1) достигается, если $A_{p}(f)>0$. Кроме того,

$$
\|f\|_{L^{p(\cdot)}}=\lambda>0 \quad \Longleftrightarrow \quad A_{p}\left(\lambda^{-1} f\right)=1 .
$$

Отсюда выводятся свойства нормы $\|\cdot\|_{L^{p(\cdot)}}$, связывающие ее с интегралом $A_{p}(f)$ :

$$
\begin{aligned}
\|f\|_{L^{p(\cdot)}} \leqslant 1 & \Longleftrightarrow \quad A_{p}(f) \leqslant 1, \\
\|f\|_{L^{p(\cdot)}} \geqslant 1 \quad \Longrightarrow & \|f\|_{L^{p(\cdot)}}^{\alpha} \leqslant A_{p}(f) \leqslant\|f\|_{L^{p(\cdot)}}^{\beta}, \\
\|f\|_{L^{p(\cdot)}} \leqslant 1 \quad \Longrightarrow & \|f\|_{L^{p(\cdot)}}^{\beta} \leqslant A_{p}(f) \leqslant\|f\|_{L^{p(\cdot)}}^{\alpha}, \\
f_{\varepsilon} \rightarrow f \text { в } L^{p(\cdot)}\left(Q_{T}\right) & \Longleftrightarrow \quad A_{p}\left(f-f_{\varepsilon}\right) \rightarrow 0 .
\end{aligned}
$$

Справедливы также следующие простые факты:

(i) имеет место непрерывное вложение пространств

$$
L^{\beta}\left(Q_{T}\right) \subset L^{p(\cdot)}\left(Q_{T}\right) \subset L^{\alpha}\left(Q_{T}\right) ;
$$

(ii) выполнено обобщенное неравенство Гёльдера

$$
\int_{Q_{T}} f g d z \leqslant 2\|f\|_{L^{p(\cdot)}\left(Q_{T}\right)}\|g\|_{L^{p^{\prime}(\cdot)}\left(Q_{T}\right)}, \quad p^{\prime}=\frac{p}{p-1} .
$$


Из (7.4) нетрудно вывести утверждение:

$$
f_{h} \rightarrow f \text { в } L^{p(\cdot)}\left(Q_{T}\right), \quad g_{h} \rightarrow g \text { в } L^{p^{\prime}(\cdot)}\left(Q_{T}\right) \quad \Longrightarrow \quad \int_{Q_{T}} f_{h} g_{h} d z \rightarrow \int_{Q_{T}} f g d z .
$$

Введенное в разделе 1 пространство $W\left(Q_{T}\right)$ рассматривается как подпространство в $L^{2}\left(Q_{T}\right) \times L^{p(\cdot)}\left(Q_{T}\right)^{n}$ с нормой

$$
\|u\|_{W\left(Q_{T}\right)}=\|\nabla u\|_{L^{p(\cdot)}\left(Q_{T}\right)^{n}}+\|u\|_{L^{2}\left(Q_{T}\right)} .
$$

Легко видеть, что $W\left(Q_{T}\right)$ замкнуто в $L^{2}\left(Q_{T}\right) \times L^{p(\cdot)}\left(Q_{T}\right)^{n}$.

Другие свойства пространств Соболева-Орлича можно почерпнуть из обширной литературы по этому вопросу, см., например, [9], [10].

7.2. Приведем доказательство теоремы 1.4, в котором ключевой оказывается

ЛЕмма 7.1. Пусть определенная на $Q_{T}$ функиия $p(z)$ удовлетворяет условиям: $p(z) \geqslant 1 u$

$$
\left|p(z)-p\left(z^{\prime}\right)\right| \leqslant \omega\left(\left|z-z^{\prime}\right|\right)
$$

əəe

$$
\omega(s)=\frac{k}{\ln (1 / s)}, \quad k=\text { const }>0, \quad s \in\left(0, \frac{1}{2}\right] .
$$

Тогда для сглаживаний $f_{h}(z)$ (см. (1.6)) справедлива оценка

$$
\int_{Q^{\prime}}\left|f_{h}(z)\right|^{p(z)} d z \leqslant c_{0} \int_{Q_{T}}\left(|f(z)|^{p(z)}+1\right) d z, \quad Q^{\prime} \Subset Q_{T},
$$

где $c_{0}=2 e^{k(n+1)}+1, h \in\left(0, h_{0}\right]$.

ДоКАЗАТЕЛЬСТВо ЛЕмМЫ 7.1. Начнем с простого наблюдения

$$
\left|f_{h}(z)\right| \leqslant h^{-(n+1)}(\sup \rho)\|f\|_{L^{1}\left(Q_{T}\right)} \equiv h^{-(n+1)} \widehat{c} .
$$

Кроме того, полагая

$$
p^{h}(z)=\min \left\{p\left(z^{\prime}\right),\left|x-x^{\prime}\right|<h,\left|t-t^{\prime}\right|<h\right\},
$$

имеем

$$
\begin{array}{rll}
1 \leqslant p^{h}(z) \leqslant p\left(z^{\prime}\right) & \text { при } & \left|x-x^{\prime}\right|<h, \quad\left|t-t^{\prime}\right|<h, \\
\lambda^{p(z)} \leqslant c_{0} \lambda^{p^{h}(z)} & \text { при } & 0 \leqslant \lambda \leqslant \widehat{c} h^{-(n+1)} .
\end{array}
$$

Для вывода $(7.8)_{2}$ заметим, что $0 \leqslant p(z)-p^{h}(z) \leqslant \omega(h)$ и $h^{-\omega(h)}=e^{k}$. Поэтому

$$
\begin{aligned}
\lambda^{p(z)} & =\lambda^{p(z)-p^{h}(z)} \lambda^{p^{h}(z)} \leqslant\left(\lambda^{\omega(h)}+1\right) \lambda^{p^{h}(z)} \\
& \leqslant\left[\left(\frac{\widehat{c}}{h^{n+1}}\right)^{\omega(h)}+1\right] \lambda^{p^{h}(z)} \leqslant c_{0} \lambda^{p^{h}(z)}, \quad h \in\left(0, h_{0}\right] .
\end{aligned}
$$

Из (7.7), (7.8) по интегральному неравенству Йенсена, получаем

$$
\begin{aligned}
& \left|f_{h}(z)\right|^{p(z)} \leqslant c_{0}\left|f_{h}(z)\right|^{p^{h}(z)} \leqslant c_{0} h^{-(n+1)} \int_{\mathbb{R}^{n+1}} \rho\left(\frac{z-z^{\prime}}{h}\right)\left|f\left(z^{\prime}\right)\right|^{p^{h}(z)} d z^{\prime}, \\
& \left|f_{h}(z)\right|^{p(z)} \leqslant c_{0} h^{-(n+1)} \int_{\mathbb{R}^{n+1}} \rho\left(\frac{z-z^{\prime}}{h}\right)\left(\left|f\left(z^{\prime}\right)\right|^{p\left(z^{\prime}\right)}+1\right) d z^{\prime} .
\end{aligned}
$$


Интегрируя (7.9) с изменением порядка интегрирования в правой части, выводим

$$
\int_{Q^{\prime}}\left|f_{h}(z)\right|^{p(z)} d z \leqslant c_{0} \int_{Q_{T}}\left(|f(z)|^{p(z)}+1\right) d z,
$$

что и требуется. Лемма доказана.

ДокАЗАТЕЛЬСТво тЕОРЕмы 1.4. Оценку (7.6) легко переписать в нормах Орлича:

$$
\left\|f_{h}\right\|_{L^{p(\cdot)}\left(Q^{\prime}\right)} \leqslant c_{1}\|f\|_{L^{p(\cdot)}\left(Q_{T}\right)}, \quad c_{1}=c_{0}\left(1+\left|Q_{T}\right|\right) .
$$

Действительно, достаточно рассмотреть случай $\|f\|_{L^{p(\cdot)}\left(Q_{T}\right)} \leqslant 1$. Тогда из (7.2) и (7.6) получим

$$
\int_{Q^{\prime}}\left|f_{h}\right|^{p} d z \leqslant c_{1}
$$

а сама оценка (7.10) следует теперь из (7.3).

Итак, сглаживания $I^{h}: L^{p(\cdot)}\left(Q_{T}\right) \rightarrow L^{p(\cdot)}\left(Q^{\prime}\right)$ равномерно ограничены по операторной норме. Поскольку $I^{h} f \rightarrow f$ равномерно в $Q^{\prime}$, если $f \in C_{0}^{\infty}\left(Q_{T}\right)$, и множество функций $C_{0}^{\infty}\left(Q_{T}\right)$ плотно в $L^{p(\cdot)}\left(Q_{T}\right)$, то из теоремы Банаха-Штейнгауза получаем сходимость сглаживающих операторов, указанную в теореме 1.4 .

\section{СПИСОК ЦИТИРОВАННОЙ ЛИТЕРАТУРЫ}

[1] J. Kinnunen, J. L. Lewis, "Higher integrability for parabolic systems of $p$-Laplacian type", Duke Math. J., 102:2 (2000), 253-271.

[2] S. Antontsev, V. Zhikov, "Higher integrability for parabolic equations of $p(x, t)$-Laplacian type", Adv. Differential Equations, 10:9 (2005), 1053-1080.

[3] О. А. Ладыженская, В. А. Солонников, Н. М. Уральцева, Линейные и квазилинейные уравнения параболического типа, Наука, М., 1967.

[4] E. DiBenedetto, Degenerate Parabolic Equations, Universitext, Springer-Verlag, New York, 1993.

[5] M. Giaquinta, G. Modica, "Regularity results for some classes of higher order nonlinear elliptic systems", J. Reine Angew. Math., 311/312 (1979), 145-169.

[6] M. Giaquinta, Multiple Integrals in the Calculus of Variation and Nonlinear Elliptic Systems, Ann. of Math. Stud, 105, Princeton Univ. Press, Princeton, NJ, 1983.

[7] F. W. Gehring, "The $L^{p}$-integrability of the partial derivatives of a quasiconformal mapping", Acta Math., 130 (1973), 265-277.

[8] В. В. Жиков, "О плотности гладких функций в пространстве Соболева-Орлича", Краевые задачи математической физики и смежные вопросы теории функиий. 35, Зап. научн. сем. ПОМИ, 310, ПОМИ, СПб., 2004, 67-81.

[9] D. E. Edmunds, J. Rákosník, "Sobolev embeddings with variable exponent", Studia Math., 143:3 (2000), 267-293.

[10] X. Fan, J. Shen, D. Zhao, "Sobolev embedding theorems for spaces $W^{k, p(x)}(\Omega)$ ", J. Math. Anal. Appl., 262:2 (2001), 749-760.

В. В. Жиков

Поступило

Владимирский государственный гуманитарный университет

22.06.2008

E-mail: zhikov@vladggu.ru

\section{С. Е. Пастухова}

Московский государственный институт радиотехники, электроники и автоматики (технический университет)

E-mail: pas-se@yandex.ru 